\title{
Occupational Therapists' Decision Making in Three Therapy Settings in Australia
}

\author{
Mehdi RASSAFIANI ${ }^{1}$ BS. OT, MS. PhD candidate, \\ Jenny ZIVIANI ${ }^{1}$ AppSc (OT), BA. MEd, PhD, \\ Sylvia RODGER ${ }^{1}$, B. OT, MEd, PhD \\ ${ }^{1}$ The University of Queensland, Brisbane, Queensland, Australia
}

\begin{abstract}
Can a work setting with its organizational, cultural, and practical considerations influence the way occupational therapists make decisions regarding client interventions? There is currently a paucity of evidence available to answer this question. This study aimed to investigate the influence of work setting on therapists' clinical reasoning in the management of clients with cerebral palsy and upper limb hypertonicity. Specifically the study aimed to examine therapists' objective and stated policies, and their intervention decisions using Social Judgement Theory methodology. Participants were 18 occupational therapists with more than five years experience with clients with cerebral palsy who were asked to make intervention decisions for clients represented by 90 case vignettes. They worked in three settings, hospitals (5), schools (6), and community (6). One participant from private setting was not included in the analysis because of lack of participants in this setting. The results indicated that therapy settings did influence therapists' decisions about intervention choices but not their objective and subjective policy decisions.
\end{abstract}

Key words: therapy setting, clinical reasoning, cerebral palsy, social judgment theory

(Asian J Occup Ther 5: 29-39, 2006)

\section{Introduction}

Clinical reasoning or thinking associated with clinical practice is the fundamental cognitive process used by therapists to plan, direct, perform, and reflect upon client care (Chapparro \& Ranka,

Received: April 30, 2006, Accepted: May 16, 2006

Corresponding to: Mehdi Rassafiani; Division of Occupational Therapy, School of Health \& Rehabilitation Sciences, The University of Queensland, Brisbane Qld 4072 Australia

phone: 617-3365-3008 fax: 617-3365-1622

e-mail:s4019578@student.uq.edu.au
2000; Schell, 1998). It has been suggested that the setting in which therapists work may influence their clinical reasoning, (Barnitt \& Partridge, 1997), facilitate or inhibit certain intervention choices (Schell \& Cervero, 1993) and consequently influence therapy outcomes for clients (Chapparo \& Ranka, 2000).

Therapy setting refers to the place in which a therapist works and provides services. The practice model adopted in different settings may, by its very nature, impact decision making. For example, therapists working in hospitals may be influenced by the medical model (Fleming, 1991a, 1991b). Furthermore, within hospital settings 
doctors can influence therapists' decision making as they retain the overall responsibility for client treatment (Crabtree \& Lyons, 1997). Subjective norms, however, can also influence therapists' decision making. Subjective norms include therapists' perceptions of the expectations of other occupational therapists, as well as those of clients and carers. These perceptions can generate a motivation to want to fulfill these expectations (Chapparo, 1997).

Barnitt and Partridge (1997) reported that therapy settings influenced experienced occupational therapists' ethical reasoning. Therapists in their study were influenced by the approval of others. In contrast, Feder, Majnemear, and Synnes (2000) did not find that work settings influenced the treatment approach chosen by 50 pediatric occupational therapists who had more than three years of clinical experience. There are two possible reasons for these discrepant findings. First, the former study examined ethical reasoning, which is a type of clinical reasoning, while the later focused on therapists' treatment choices that are the end product of therapists' decision making processes. Second, the studies employed different research methods. While Barnitt and Partridge (1997) employed a phenomenological method relying on observation and interview, Feder, Majnemear, and Synnes (2000) used a quantitative approach based on a questionnaire.

To date no studies have attempted to determine the effects of therapy settings on different aspects of therapists' decision making such as the information used by therapists, and the intervention options recommended for their clients. Understanding what information is used by therapists in their decision making in different settings can help identify the specific assessment and evaluation procedures considered important and result in more effective use of time. If intervention strategies chosen by occupational therapists for their clients vary on the basis of clinical setting then it also brings into question the issue of best practice. Social Judgment Theory (SJT) method seems to be appropriate in studying information used and intervention choices employed by occupational therapists in their decision making.
Social Judgment Theory (SJT) is a cognitive decision making theory (Doherty \& Kurz, 1996) which is most relevant in situations where no optimal decision exists (Cooksey, 1988). There are two assumptions underlying the nature of decision makers' thinking processes in SJT. First, any decision must be based on pieces of information or factors (Cooksey \& Freebody, 1986). Second, decision makers integrate these factors and synthesize them, trade off factors against one another, set limits for factors, select and attend to only those factors having perceived importance to arrive at their final decisions (Hammond, 1978, 1981 cited in Cooksey \& Freebody, 1986). In other words, decision makers are influenced by the specific features of the problem confronting them. In this process they differentially weigh factors in their clinical reasoning and this is called strategy or policy capturing (Wigton, 1996). This process is not entirely analytical, that is applying mathematically logical rules, but largely intuitive based on clinicians' hunches, impressions, and feelings (Hammond, 1996). Two kinds of policies are identified in SJT: objective (statistical) and stated (subjective) policies (Cooksey, 1996a). Objective policy is based on intuitive aspects of cognition and refers to identified levels of importance attached to the factors or how therapists actually use factors (information) in their decision making. Objective policies are identified when a large number of case vignettes or real cases are developed and presented to the decision makers who are then required to respond to a clinical question. General linear analysis is used to analyse the relationship between the factors (information) presented in the cases and the person's response and this determines what has been called the statistical factor weights (Cooksey, 1996b). Stated policies, on the other hand refer to an individual's stated impressions of levels of importance (weights) attached to factors or how they think they use factors (information) when they make decisions. It is based on the analytical aspects of cognition and identified when the factors are presented to the decision makers who are then asked to identify a factor's weight (e.g., by factor ranking). In the present study, we 


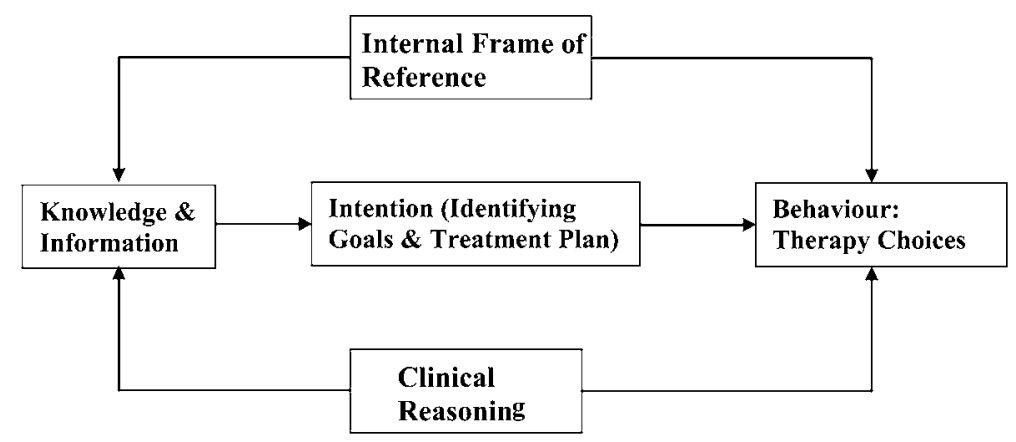

Fig. 1. A simple model in clinical reasoning (Chapparo, 1997)

examine the impact of therapy settings on therapists' decision making in the management of clients with cerebral palsy and upper limb hypertonicity in terms of therapists' objective and stated factor weights, as well as therapists' intervention choices.

The management of upper limb hypertonicity of clients with cerebral palsy (CP) was chosen as the focus of this study for two main reasons. First, the incidence of $\mathrm{CP}$ is high, manifesting in between 1.4 and 2.4 of 1000 live births (Parkes, et al. 2001; Pirpiris \& Graham, 2001; Sanger, 2003). Second, people with CP are one of the major client groups referred to occupational therapy clinics as outpatients (Dudgeon, 2001).

Chaparro's frame of reference (1997) is used to further clarify the aims of this study. She suggested a model for therapists' clinical reasoning within a personal internal frame of reference. She proposed that knowledge related to context, clients, other professionals, intervention as well as self-beliefs and professional beliefs produced the knowledge content of clinical decision making and these all form a complex personal internal frame of reference. Therapists use this knowledge within the internal frame of reference to build a "personal theory for each clinical encounter. She suggested that this frame of reference manages clinical judgments. Figure 1 illustrates Chapparo's model in which the relationship between the internal frame of reference and clinical reasoning is shown. This study focused on weights attributed to knowledge and information (factors) in two ways including objective and stated policies, and therapists' intervention choices.

\section{Method}

This study used the capacity of SJT to investigate and understand the impact of therapy settings on individual therapists' decision making policies and their intervention choices in the management of upper limb hypertonicity in clients with CP.

\section{Task development}

On the basis of a comprehensive literature review, discussion with two expert therapists, and a survey of 12 further experienced clinicians, 12 factors were considered to be influential when therapists make decisions in the management of upper limb hypertonicity of clients with CP (see Table 1). These factors were the basis for the development of case vignettes used in this study. First, factors were defined and measurement scales for each determined. The 12 identified factors and their scales of measurement formed the basis of the information used to develop 10 sample case vignettes. A manual containing instructions for answering the case vignettes, as well as the factor definitions and the scales of measurement was also developed to provide appropriate information for therapists. The manual included three sections. The first section provided information about the process of identifying the factors. The second section included explanations about the identified factors and their scales of measurement. The last 
section comprised a sample case vignette and study questions (Rassafiani, 2006).

Therapists were asked to identify the most appropriate treatment method for the client represented in the case vignettes among seven choices that were ordered on the basis of how invasive they might be considered: no therapy, hands-on approach, splinting, constraint-induced movement therapy, casting, refer for Botulinum Toxin Type A (BTX-A), and refer for surgery. These intervention options were based on the work of Copley and Kuipers (1999).

A pilot study was conducted with four experienced occupational therapists to determine: (1) whether the administration manual (i.e., the factor definitions, and the scales of measurement) was readily understood; (2) if the sample case vignettes were perceived as realistic; and (3) the time requirement for reading the manual and answering case vignettes was acceptable. The pilot study enabled the administration manual and case vignettes to be refined enabling the next phase to proceed which was the generation of case vignettes for this study.

The statistical program SPSS version 11 was used to help generate random values for each of the factors included in the case vignettes. In generating the data, a uniform distribution was chosen for each factor in which the values ranged from the lowest to the highest to make sure that there would be a wide spread in the final selected case vignettes mimicking the real world. Four hundred case profiles were generated and these were then reviewed by two occupational therapists experienced in the management of upper limb hypertonicity as well as the research team to exclude implausible client profiles. From the original 400, 90 case vignettes that were considered to be representative of real clients (see Appendix A for an example). This was considered a reasonable numbers of case vignettes to use in judgment analysis (Cooksey, 1996a).

All chosen case vignettes were collated into a booklet. The booklet was bound and accompanied by the manual guiding the participants in how to answer case vignettes. Moreover, the manual contained explanations and definitions of the factors along with their scales of measurement.

\section{Procedure}

Ethical approval was gained from The Behavioural and Social Sciences Ethical Review Committee (BSSERC), The University of Queensland. All questionnaires, answer sheets, and demographic information were labelled with appropriate codes related to participants and kept in a locked filing cabinet. When a participant agreed to take part in the study, an appointment was made for two and half hours at her/his convenience. During the meeting the participants were told about the study and the process of identifying the factors, their scales of measurement, and their definitions, as well as development of the case vignettes. Then, they were asked to read the manual and make interventions choices for three example case vignettes. In the meantime, any queries regarding the various aspects of the study were clarified. They were then provided with the case vignettes booklet and an answer sheet and asked to complete 90 case vignettes. They could refer to the manual when they were answering the questions. Refreshments were provided during a break after they completed the first 60 case vignettes. When they finished all the case vignettes they were asked to rank in order of priority the 12 identified factors of the study. These factors were written on individual cards which participants could manipulate until they were satisfied with the order.

\section{Participants}

Occupational therapists with more than five years experience with clients with $\mathrm{CP}$ working in central and south-east Queensland (Australia) were invited to participate in this study. They had to satisfy the following criteria: (a) have a minimum qualification of a Bachelor of Occupational Therapy; (b) possess additional formal training (such as workshops or courses on $\mathrm{CP}$ ); and (c) be acknowledged by professional colleagues as having experience in the management of upper limb hypertonicity in children and adults.

Participants were recruited by advertising in the Occupational Therapy Australia QLD Newsletter; distributing flyers about the project to paediatric interest groups; and by snowball 
sampling. Eighteen occupational therapists (16 females and 2 males), mean age of 36.7 years $(\mathrm{SD}=7.6$ years) who had worked with people with $\mathrm{CP}$ for a mean of 10.4 years $(\mathrm{SD}=6.0$ years $)$ participated. All possessed a Bachelor degree in occupational therapy and one had a PhD. On average, the participants had attended 5.8 $(\mathrm{SD}=3.1)$ continuing education workshops (e.g., NDT, sensory integration, and conductive education). Participants worked in various settings including: (a) five in hospitals; (b) six in schools; and (c) six in the community. One participant was from the private sector and was not included in the subsequent analysis.

\section{Statistical Methods}

Data from the answer sheet and therapists' decisions for the 90 case vignettes were analysed using Chi-square test to determine whether the therapists' work setting (i.e., Hospital, school, or community) influenced the intervention choices favoured. Multiple linear regression models were employed to examine therapists' objective policies. To examine the relative importance of the factors the square semi-partial correlations for 12 factors resulting from multiple linear regression models were used and the relative weighting of the factors were identified by the following equation.

$$
\mathrm{RW}_{\mathrm{ui}}=\frac{\mathrm{sr}^{2}}{\Sigma \mathrm{sr}^{2}}
$$

RW is the objective weight and $\mathrm{sr}^{2}$ is the square semi-partial correlation resulting from the linear regression coefficient (Cooksey, 1996a). The results show how therapists have used factors to decide about the management of upper limb hypertonicity for clients with $\mathrm{CP}$. In addition, to examine stated relative weights of factors influencing therapists' decision making, the SMARTER (Simple Multi-Attribute Rating Technique Extended to Ranking) procedure was employed (Barron \& Barrett, 1996). Participants ranked the twelve judgment factors used in the study from 1 to 12 with 1 being the most important and 12 the least. Then, by using the SMARTER, stated weights were calculated for each factor. Finally, One-way Analysis of Variances
(ANOVA) was carried out to determine whether there was any significant difference in therapists' objective and stated policies in respect to their work settings.

\section{Results}

\section{Intervention choices}

Figure 2 reports the percentage of therapists who chose specific interventions based on their work settings. The Chi-square test showed that therapists' treatment choices were significantly different $\left(\chi^{2}=399.9, \mathrm{df}=12 ; \mathrm{p}=0.000\right)$.

Inspection of Fig. 2 reveals that hands-on techniques, splinting, casting, and referral for BTX-A injection were the main treatment choices within all settings. While hands-on techniques and splinting were the most commonly chosen intervention methods for participants working in the school settings, splinting and casting were mainly chosen by participants in community settings. Intervention choices favoured casting and referral for BTX-A for participants working in hospitals. Intervention methods including notherapy, CIMT, and referral for surgery were chosen less frequently than other methods and their percentages among the three groups of participants were comparable.

To further clarify the differences in treatment choices depending on settings, CIMT was removed from the analysis. CIMT was among the least favoured treatment options and this may be reflective of its clinical novelty. Figure 3 illustrates the treatment choices after removal of the CIMT option. The treatment choices for therapists practicing at schools were hands on techniques and splinting, this shifted to casting for therapists working in the community setting and referral for BTX-A injections for those working in hospitals. These differences were statistically significant $\left(\chi^{2}=384.1, \mathrm{df}=10, \mathrm{p}=0.000\right)$.

\section{Therapists' objective and stated policies}

The objective polices of the participants working in the three therapy settings were then compared using ANOVA. The results revealed no statistically significant differences among groups in relative weights for the 12 factors of the study. 


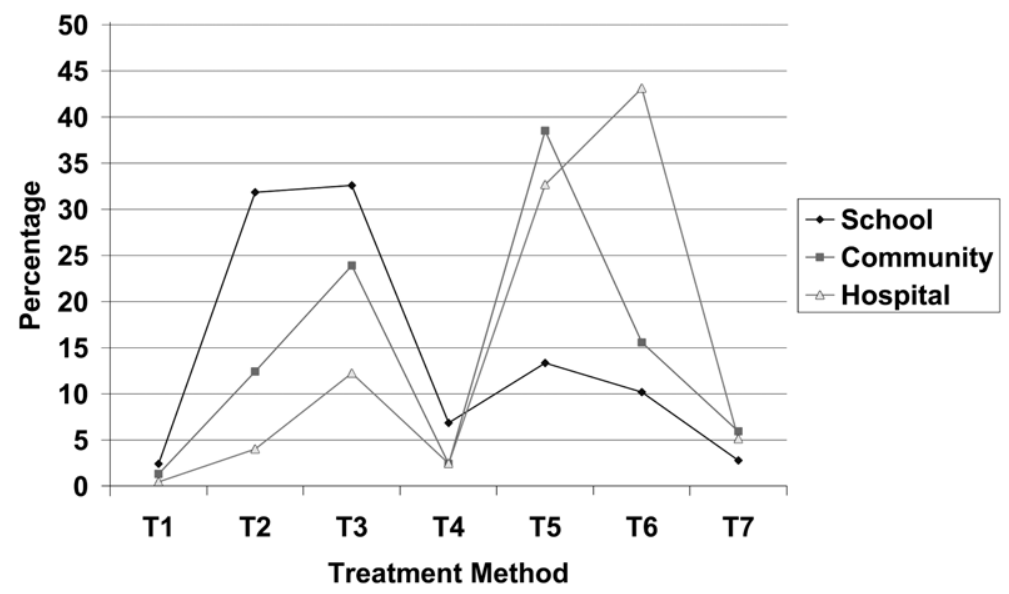

Fig. 2. The frequency of treatment methods chosen by therapists based on work settings

Note. T1: No therapy; T2: Hands-on techniques; T3: Splinting; T4: Constraint-induced movement therapy (CIMT); T5: Casting; T6: Referral for BTX-A injection; T7: Referral for surgery.

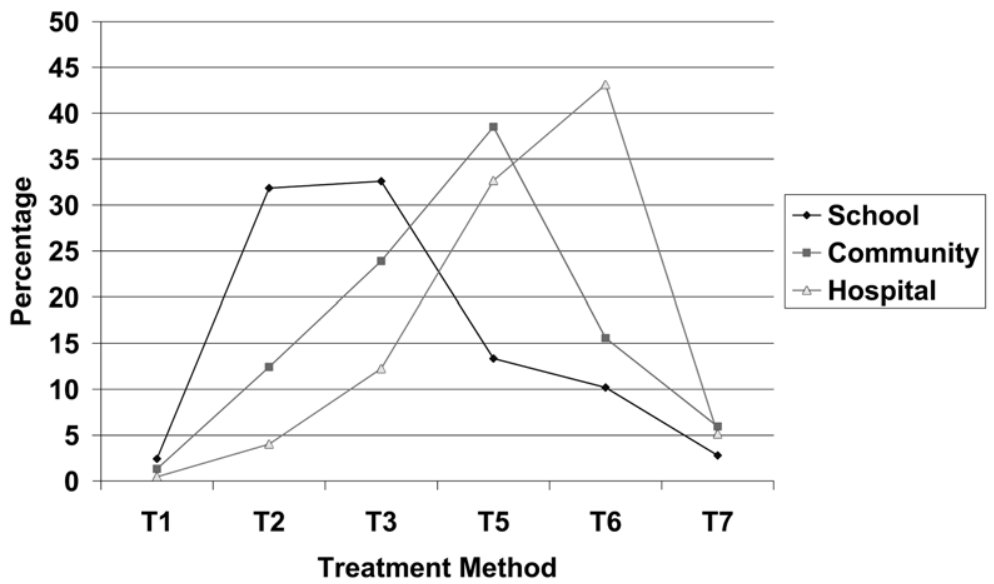

Fig. 3. The frequency of treatment choices by therapists in three setting with removal of constraint-induced movement therapy as an option Note. T1: No therapy; T2: Hands-on techniques; T3: Splinting; T5: Casting; T6: Referral for BTX-A injection; T7: Referral for surgery.

The $p$ values for all factors were more than the critical value of $0.05(\mathrm{df}=2,14)$. Table 1 summarizes these results.

ANOVA was used to examine whether there was any difference in the stated relative weights of factors among the three groups. Results indicated no statistically significant difference among the three groups. The $p$ values ranged between 0.2 and 0.99 for the twelve factors (Table 2). 
Table 1. Comparison of objective policies of therapists working in three work settings

\begin{tabular}{lcccccccc}
\hline & \multicolumn{2}{c}{ Community } & \multicolumn{2}{c}{ School } & \multicolumn{2}{c}{ Hospital } & & \\
\cline { 2 - 6 } Factor & Mean & SD & Mean & SD & Mean & SD & & $F$ \\
\hline Age & 5.7 & 5.8 & 18.5 & 23.4 & 1.4 & 1.7 & 2.1 & 0.15 \\
PT $^{1}$ & 4.5 & 3.7 & 3.5 & 4.1 & 12.2 & 12.6 & 2.1 & 0.15 \\
OG $^{2}$ & 10 & 20.2 & 1.7 & 2.4 & 2.2 & 2.2 & 0.9 & 0.45 \\
SS $^{3}$ & 15.3 & 16 & 23.5 & 28.6 & 45.8 & 13.7 & 3 & 0.08 \\
LP $^{4}$ & 2.3 & 3.1 & 2.8 & 2.1 & 5 & 5.6 & 0.8 & 0.49 \\
LA $^{5}$ & 4.2 & 5.7 & 6.3 & 9.6 & 1.2 & 1.6 & 0.8 & 0.47 \\
LD $^{6}$ & 2 & 2.4 & 1.8 & 1.6 & 5.6 & 5.9 & 1.8 & 0.2 \\
WFP $^{7}$ & 10.8 & 7.2 & 23 & 31.8 & 4 & 3.8 & 1.3 & 0.29 \\
TP $^{8}$ & 4 & 5.1 & 2.5 & 1.9 & 11.2 & 9.3 & 3.3 & 0.07 \\
PC $^{9}$ & 5.3 & 5.7 & 3.3 & 3.6 & 5.2 & 6.1 & 0.3 & 0.77 \\
AM $^{10}$ & 16 & 14.4 & 5.2 & 8.8 & 4.8 & 6.7 & 2 & 0.17 \\
FB $^{11}$ & 19.5 & 24.4 & 7.8 & 11.6 & 1 & 1.4 & 1.9 & 0.19 \\
\hline
\end{tabular}

Note. ${ }^{1}$ Previous Treatment; ${ }^{2}$ Occupational Goals; ${ }^{3}$ Severity of Spasticity; ${ }^{4}$ Limitation in Passive Range of Movement; ${ }^{5}$ Limitation in Active Movement; ${ }^{6}$ Limitations in Developmental Gross Movement; ${ }^{7}$ Wrist and Finger Posture; ${ }^{8}$ Thumb Posture; ${ }^{9}$ Practice Context (time limitation, treatment priority); ${ }^{10}$ Associated Medical Factors; ${ }^{11}$ Client \& Family Background.

Table 2. Comparing stated policies of therapists working in three therapy settings

\begin{tabular}{|c|c|c|c|c|c|c|c|c|}
\hline \multirow[b]{2}{*}{ Factor } & \multicolumn{2}{|c|}{ Community } & \multicolumn{2}{|c|}{ School } & \multicolumn{2}{|c|}{ Hospital } & \multirow[b]{2}{*}{$F$} & \multirow[b]{2}{*}{$p$} \\
\hline & Mean & SD & Mean & SD & Mean & $\mathrm{SD}$ & & \\
\hline Age & 8.1 & 6.3 & 12.1 & 11.1 & 8.3 & 5.4 & 0.46 & 0.64 \\
\hline $\mathrm{PT}^{1}$ & 5.6 & 4.7 & 5.3 & 4.1 & 5.5 & 3.4 & 0.01 & 0.99 \\
\hline $\mathrm{OG}^{2}$ & 16.7 & 8 & 18.3 & 7.5 & 16.9 & 8.4 & 0.07 & 0.93 \\
\hline $\mathrm{SS}^{3}$ & 13.8 & 7 & 14 & 6.6 & 15.2 & 7.8 & 0.07 & 0.94 \\
\hline $\mathrm{LP}^{4}$ & 7.5 & 3.4 & 10.4 & 8.9 & 11.3 & 5.1 & 0.57 & 0.58 \\
\hline $\mathrm{LA}^{5}$ & 9.7 & 3.4 & 9 & 6.8 & 8.3 & 3.2 & 0.11 & 0.89 \\
\hline $\mathrm{LD}^{6}$ & 2.6 & 2 & 2 & 1.9 & 1.7 & 1.1 & 0.43 & 0.66 \\
\hline $\mathrm{WFP}^{7}$ & 4.6 & 3.4 & 8.5 & 6.1 & 4 & 1.7 & 1.9 & 0.19 \\
\hline $\mathrm{TP}^{8}$ & 3.8 & 2.8 & 5.2 & 2.6 & 4.6 & 1.5 & 0.53 & 0.6 \\
\hline $\mathrm{PC}^{9}$ & 3 & 2.3 & 6 & 2.6 & 6.5 & 10.8 & 0.54 & 0.59 \\
\hline $\mathrm{AM}^{10}$ & 13.2 & 11.3 & 3.3 & 2.4 & 7.6 & 10.7 & 1.84 & 0.2 \\
\hline $\mathrm{FB}^{11}$ & 11.7 & 10 & 6.1 & 4.8 & 10.2 & 7 & 0.86 & 0.44 \\
\hline
\end{tabular}

Note. ${ }^{1}$ Previous Treatment; ${ }^{2}$ Occupational Goals; ${ }^{3}$ Severity of Spasticity; ${ }^{4}$ Limitation in Passive Range of Movement; ${ }^{5}$ Limitation in Active Movement; ${ }^{6}$ Limitations in Developmental Gross Movement; ${ }^{7}$ Wrist and Finger Posture; ${ }^{8}$ Thumb Posture; ${ }^{9}$ Practice Context (time limitation, treatment priority); ${ }^{10}$ Associated Medical Factors; ${ }^{11}$ Client \& Family Background.

\section{Discussion}

Participants in this study were employed in one of three clinical settings - hospital, school, or community. When asked to identify influential factors in their decision making therapists regardless of work settings chose similar factors.
Therapists in the three work settings were also similar in their objective policies. However, even though they identified similar factors as being influential they varied in how they used this information when determining the intervention choices for clients represented in the case vignettes. 
The most frequently identified interventions by participants working in school settings were hands-on techniques and splinting. For therapists working in community settings, splinting and casting were the primary choices, while for those working in hospitals, casting, and referral for BTX-A injection were preferred. Paradoxically, however, these variations did not significantly influence therapists' objective and stated policies. Ryan (2000) has stated that the focus of health care is predominantly intervention and medical strategies in hospitals and that this changes towards prevention and social ways of working in community and school settings. Therefore, different thinking and clinical reasoning for these settings is required. On the basis of studies in medicine and nursing, she further divided aspects of clinical reasoning into generalized (e.g., interpersonal skills, technical skills, selection of data) and specific (e.g., problem solving, number of hypothesis, physical examination) to the setting. The findings of this study seem to support Ryan's (2000) proposition.

The results of this study are in contrast to those of Feder, Majnemear, and Synnes (2000) in which they concluded that work settings did not influence choice of treatment approach for 50 pediatric occupational therapists with more than three years of experience. This might be due to the differences in the methods of these studies. Both studies employed quantitative methods, however, this study used case vignettes to explore the intervention options, but Feder et al. employed a questionnaire asking directly about intervention methods.

Several reasons might help explain the various interventions used by therapists in different clinical settings. Chapparo (1997) stated that "therapists were less likely to participate in therapy that was perceived by them to be 'difficult' or 'impossible' in their particular work context” (p.333). Participants working in hospitals could have been more influenced by the medical context of their setting (Mattingly, 1994). Fleming (1991a, 1991b) suggested two other reasons why therapists are strongly influenced by the medical model: the type of education they have received, and their exposure to medical lectures and case studies. Occupational therapy education may therefore encourage therapists to employ the medical model. This may have been the case particularly for therapists who trained more than five years ago. Moreover, the medical model is easy to understand as it includes concrete information and therapists can follow the procedures in a step by step method; such a model is used in scientific reasoning (Chapparo \& Ranka, 2000). Therapists working in hospitals also usually take part in lectures and case discussions run by physicians such as in grand rounds. This may also increase their knowledge about medical interventions and consequently influence their own intervention choices.

The concept of subjective norms is another influential factor in clinical decision making (Chapparo, 1997). Subjective norms refer to "the perceived social pressure to perform or not to perform various forms of therapy" (Chapparo, 1997: p. 279). Subjective norms evolve from: (1) therapists' perceptions of the expectations of other occupational therapists, and (2) therapists' perceptions of clients' and carers' expectations and can result in a motivation to fulfill these expectations. In this research, subjective norms relating to specific settings may have influenced therapists in choosing particular intervention options. In the schools, occupational therapists mostly had interactions with teachers, physical therapists and speech and language pathologists, while in hospitals this extended to nurses, and doctors. In each setting, therefore different professionals had various expectations and could potentially influence therapists' choice of interventions. Finally, difference in therapists' intervention choices may not be related to therapy settings but therapists may simply choose to work in settings consistent with their view of health care, or a combination of these factors may cause differences.

\section{Limitations and future research}

For the participants in this study place of employment impacted intervention choices. This means that for the same client, different clinical decisions were made if therapists were in different settings. In other words, if a client obtained advice 
from an occupational therapist in a school setting, this might be different from the intervention advice of a therapist who worked in a hospital setting. If a client goes to different settings and receives different suggestions, this may produce conflict in choosing the most appropriate intervention methods. However, because of the small numbers of participants in this study this issue requires further investigation to examine what currently happens with those clients who are referred to different settings.

This study was conducted with the case vignettes. Further investigation with real clients would help to clinically understand the influence of therapy setting on therapists' decisions.

\section{Conclusion}

This study found that therapists' intervention strategies were influenced by the therapy setting in which they were employed. In contrast, objective and stated policies remained statistically similar across settings. These findings suggest that different processes are at work in the clinical decisions made by therapists depending on their place of employment. Understanding the impact of therapy settings on therapists' decision making is an important consideration when looking at best practice. In this study therapists in three settings used different intervention options for clients. It is necessary to do further study with therapists in other settings and investigate the influences of therapy settings on all aspect of therapists' clinical reasoning.

\section{Acknowledgment}

The authors would like to thank all therapists who participated in various phases of this study. Appreciation is extended to Dr Jodie Copley and Kathy Kuipers for their expertise in the area of upper limb hypertonicity.

\section{References}

Barnitt, R., \& Partridge. (1997). Ethical reasoning in physical therapy and occupational therapy. Physiotherapy Research International, 2(3), 178192.
Barron, F. H., \& Barrett, B. E. (1996). The efficacy of SMARTER - simple multi-attribute rating technique extended to ranking. Acta Psychologica, 93, 23-36.

Chapparo, C. (1997). Influences on clinical reasoning in occupational therapy. Unpublished $\mathrm{PhD}$, Sydney, Australia: Macquarie University.

Chapparo, C., \& Ranka, J. (2000). Clinical reasoning in occupational therapy. Higgs J. \& Jones M. (eds.), Clinical reasoning in health professions (pp. 128-137). Oxford: Butterworth Heinemann.

Cooksey, R. W. (1988). Social judgment theory in education: current and potential applications. Brehmer B. \& Joyce C. R. B. (eds.), Human judgment: the SJT view (pp. 273-315). Amsterdam: North-Holland.

Cooksey, R. W. (1996a). Judgment analysis: theory, method, and applications. San Diego: Academic press.

Cooksey, R. W. (1996b). The methodology of social judgment theory. Thinking and Reasoning, 2(2/3), 141-173.

Cooksey, R. W., \& Freebody, P. (1986). Social judgment theory and cognitive feedback: a general model for analyzing educational policies and decisions. Educational Evaluation and Policy Analysis, 8(1), 17-29.

Copley, J., \& Kuipers, K. (1999). Management of upper limb hypertonicity. San Antonio, TX: Therapy Skill Builders.

Crabtree, M., \& Lyons, M. (1997). Focal points and relationships: a study of clinical reasoning. $\mathrm{Br} . \mathrm{J}$. Occup. Ther., 60(2), 57-64.

Doherty, M. E., \& Kurz, E. M. (1996). Social judgment theory. Thinking and Reasoning, 2, 109-114.

Feder, K., Majnemer, A., \& Synnes, A. (2000). Handwriting: Current trends in occupational therapy practice. Can. J. Occup.Ther., 67(3), 197204.

Fleming, M. H. (1991a). Clinical reasoning in medicine compared with clinical reasoning in occupational therapy. Am. J. Occup. Ther., 45(11), 988-996.

Fleming, M. H. (1991b). The therapist with the threetrack mind. Am. J. Occup. Ther., 45, 1007-1014.

Hammond, K. R. (1996). Human judgment and social policy: irreducible uncertainty, inevitable error, unavoidable injustice. New York: Oxford University Press.

Mattingly, C. (1994). Occupational therapy as a twobody practice: body as machine. Mattingly C. \& 
Fleming M. H. (eds.), Clinical reasoning: forms of inquiry in a therapeutic practice. (pp. 37-63). Philadelphia: FA Davis.

Rassafiani, M. (2006). Occupational therapists, decisions about the management of upper limb hypertonicity in children and adolescents with cerebral palsy. Unpublished PhD, Brisbane: University of Queensland.

Ryan, S. (2000). Facilitating the clinical reasoning of occupational therapy students on fieldwork placement. Higgs J. \& Jones M. (eds.), Clinical reasoning in the health professions ( 2 nd ed., pp.
242-248). Oxford: Butterworth-Heinmann.

Schell, B. B. (1998). Clinical reasoning: the basis of practice. Neistadt M. E. \& Crepeau E. B. (eds.), Willard and Spackman's occupational therapy (9th ed., pp. 90-100). Philadelphia: LippincottRaven Publishers.

Schell, B. B., \& Cervero, R. M. (1993). Clinical reasoning of occupational therapy: an integrative review. Amer. J. Occup. The., 47, 605-610.

Wigton, R. S. (1996). Social Judgment theory and medical judgement. Thinking and Reasoning, 2(2/ 3), 175-190. 


\section{Appendix A - Example of case vignette}

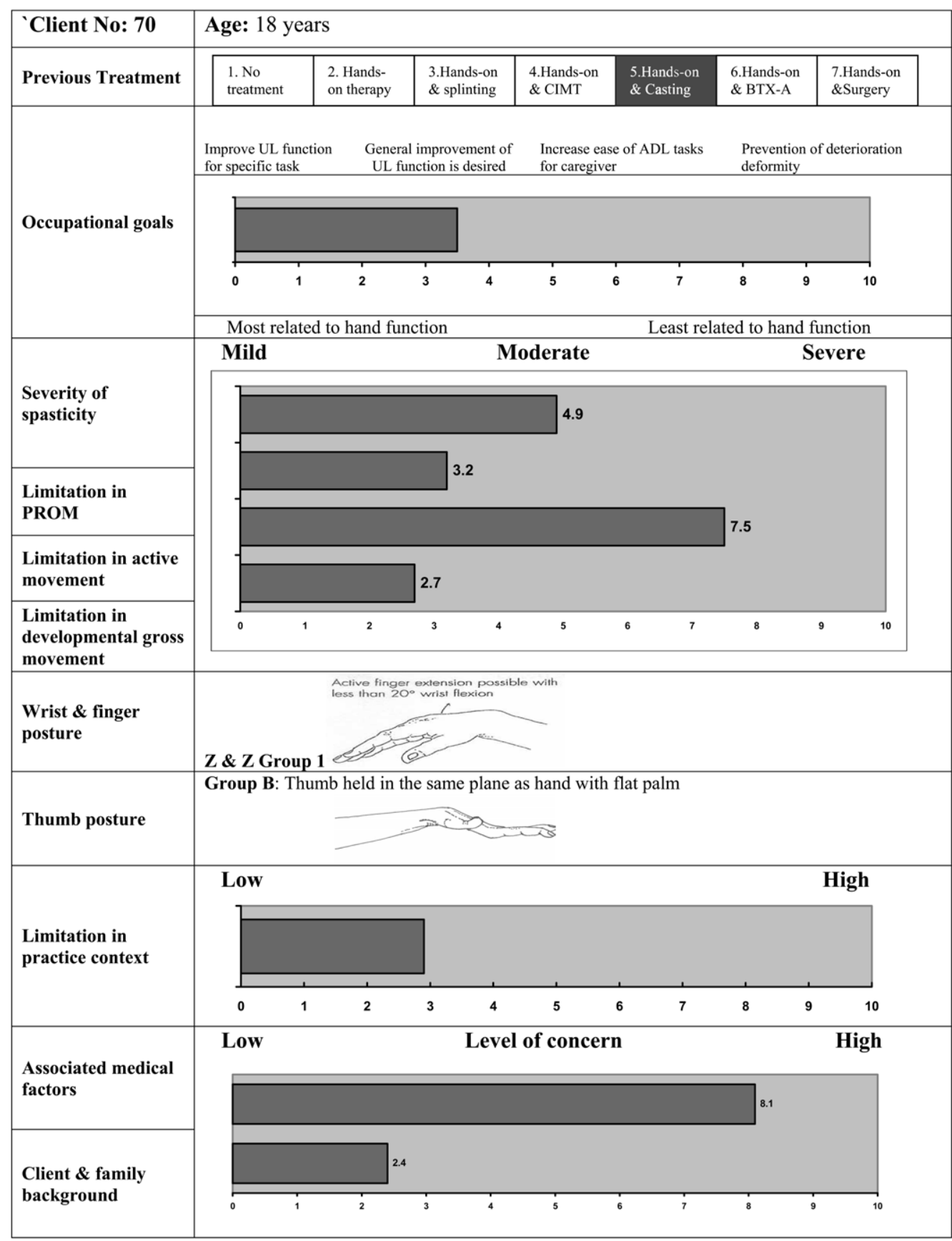

